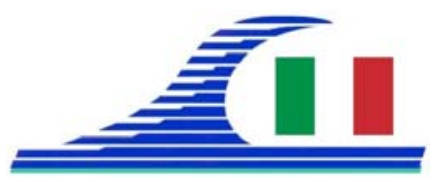

Conférence Méditerranéenne Côtière et Maritime EDITION 3, FERRARA, ITALIA (2015)

Coastal and Maritime Mediterranean Conference

Disponible en ligne - http://www.paralia.fr - Available online

\title{
Cartographie du fond marin par les méthodes de sismique réflexion et du sonar latéral
}

\section{Souad BATATA SABETE ${ }^{1}$}

1. Laboratoire d'Etudes Maritimes LEM / Centre des Etudes sur Site

Djenane El Malek BP 848 Hydra, Alger, Algérie.

sabetegeoph@yahoo.fr

\section{Résumé :}

Cette étude rentre dans le cadre du projet de réalisation d'une prise d'eau et l'extension de la centrale électrique de Cap Djinet. La cartographie du fond marin de ce site s'appuie essentiellement sur l'exploitation des données de sismique réflexion et de celles de la campagne géophysique de sonar latéral ainsi que sur une étude géologique basée sur une synthèse bibliographique d'observations de terrain et de sondages géotechniques (CHOUAKI, 1981). Les données ainsi collectées ont servi à l'établissement d'une carte bathymétrique et d'une carte géomorphologique.

Mots-clés : Cartographie, Fond marin, Sismique réflexion, Sonar latéral, Géologie, Bathymétrie, Géomorphologie.

\section{Introduction}

La région de "Cap Djinet" est une petite ville côtière algérienne, située dans la Wilaya de Boumerdès, plus précisément entre l’oued Laâmara et l’oued Isser (figure 1).

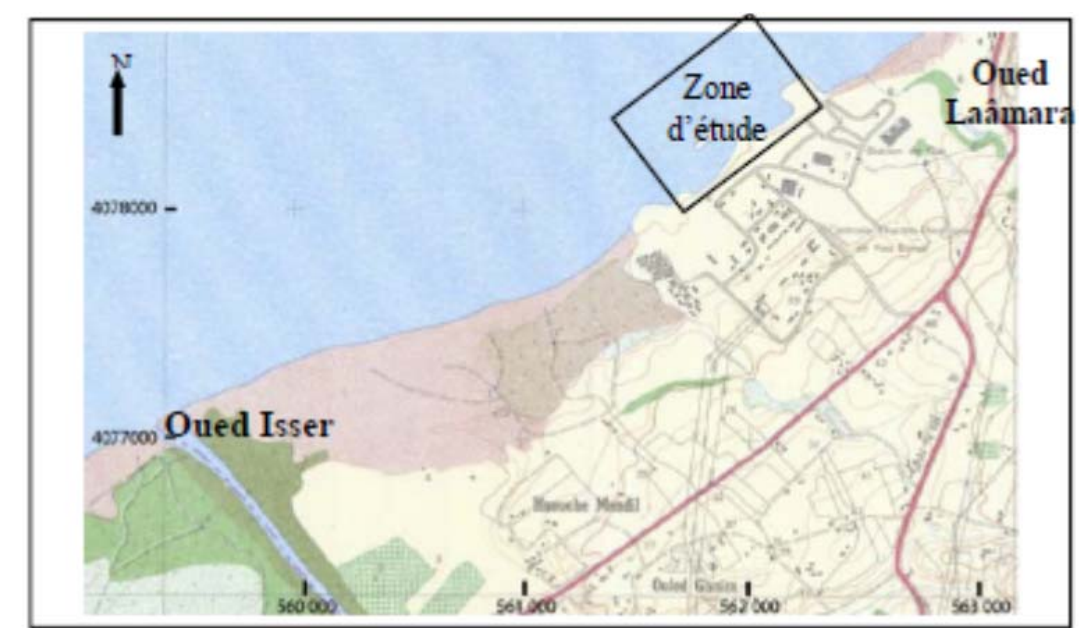

Figure 1. Extrait de la carte topographique de Dellys au 1/25 000.

La géologie de cette localité se distingue par une lithologie variée caractérisée par des dépôts sédimentaires du Mio-Plio-Quatenaire et un volcanisme basaltique. (FICHEUR 1895 ; GLANGEAUD et al., 1952). 
Côtes méditerranéennes menacées :

Risques et défis dans le contexte du changement climatique

La sismique réflexion permet de visualiser les structures géologiques en profondeur, grâce à l'analyse des échos d'ondes acoustiques émises par un transducteur.

Le sonar latéral est un émetteur-récepteur d'ondes acoustiques permettant d'obtenir des informations sur la morphologie et la nature texturale du fond marin.

Le présent document décrit la nature des travaux réalisés à travers la zone d'étude, les moyens mis à disposition, les méthodes utilisées ainsi que données ayants servi à la conception des documents cartographiques.

\section{Matériels et méthodes}

\subsection{Matériels}

Les travaux d'acquisition se sont déroulés en juin 2012, et ont consisté au levé de 25 km de profils sismiques et de $22 \mathrm{~km}$ de profils sonar.

Les moyens matériels utilisés sont une chaine sismique IXSEA type Echoes 1,5 kHz, embarquée à bord d'une vedette, une flûte d'hydrophones et un sonar à balayage latéral C-MAX 2, composé d'un poisson remorqué à une vitesse d'environ 4 nœuds.

Le positionnement des profils sismiques est assuré par un système GPS en mode RTK (real time kinematic).

Le traitement des données brutes s'est effectué au moyen des logiciels DELPH sismique et HY"PACK.

\subsection{Méthodes}

- Positionnement sur carte des profils sismiques et des profils sonar.

- Reconnaissance de la zone d'étude.

- Réalisation d'un profil d'essai, de contrôle et de calibrage du transducteur.

- Contrôle de la qualité des données numériques (fréquence et amplitude).

- Collecte en temps réel des données brutes.

- Vérification et traitement des données brutes.

- Digitalisation des réflecteurs.

- Interprétation des profils sismiques et de l’imagerie acoustique (mosaïque des faciès).

- Corrélation des sections sismiques.

- Corrélation entre les données sismiques et les données du sonar latéral.

- Cartographie et interprétation de la carte bathymétrique.

\section{3- Résultats}

Les principaux résultats de cette étude sont :

- Identification grâce à la sismique réflexion des réflecteurs, le premier représente le fond marin, le deuxième est une discontinuité dans les sables et le troisième marque une transition sables-marnes et représente le substratum (figure 2).

- Etablissement d’une carte bathymétrique du fond marin (figure 3). 
- Etablissement grâce au sonar latéral d'une cartographie des faciès acoustiques identifiés sur la carte géomorphologique (figure 4).

- Estimation de l'épaisseur des sables à travers l'analyse des carottes de sondage.

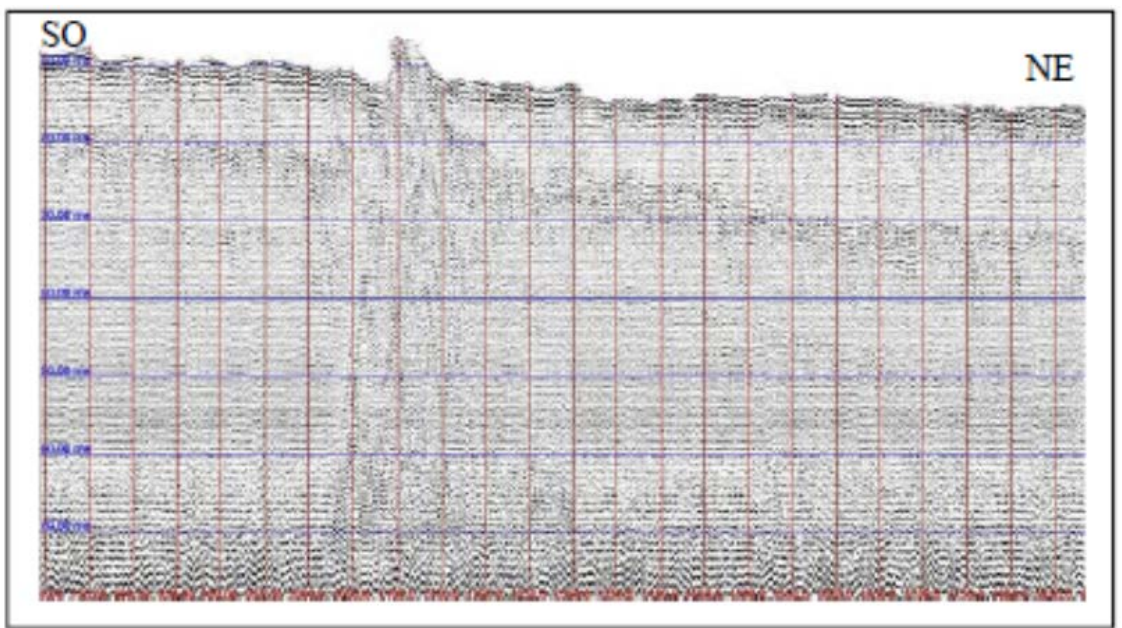

Figure 2. Section sismique.

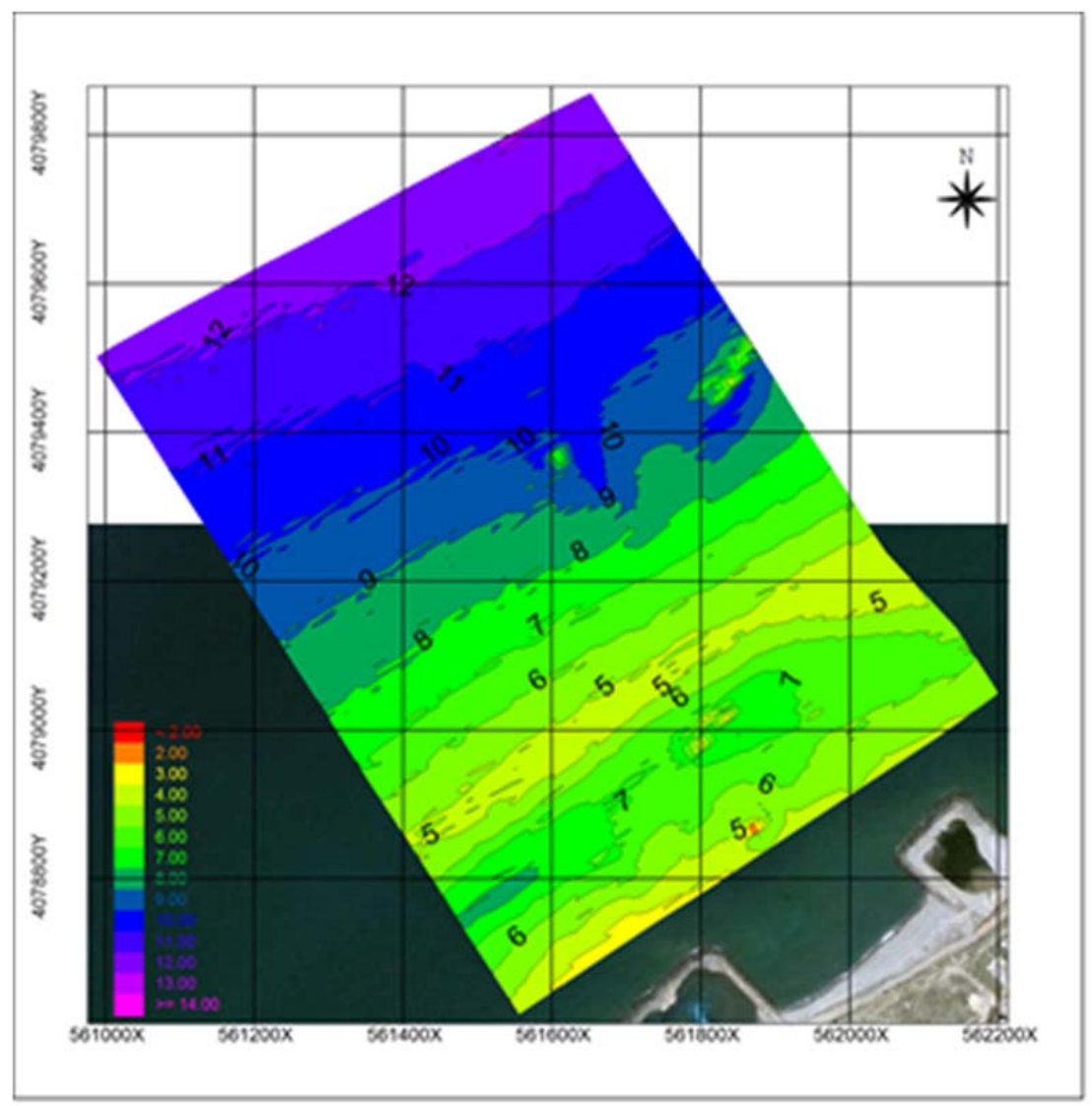

Figure 3. Carte bathymétrique. 
Côtes méditerranéennes menacées :

Risques et défis dans le contexte du changement climatique

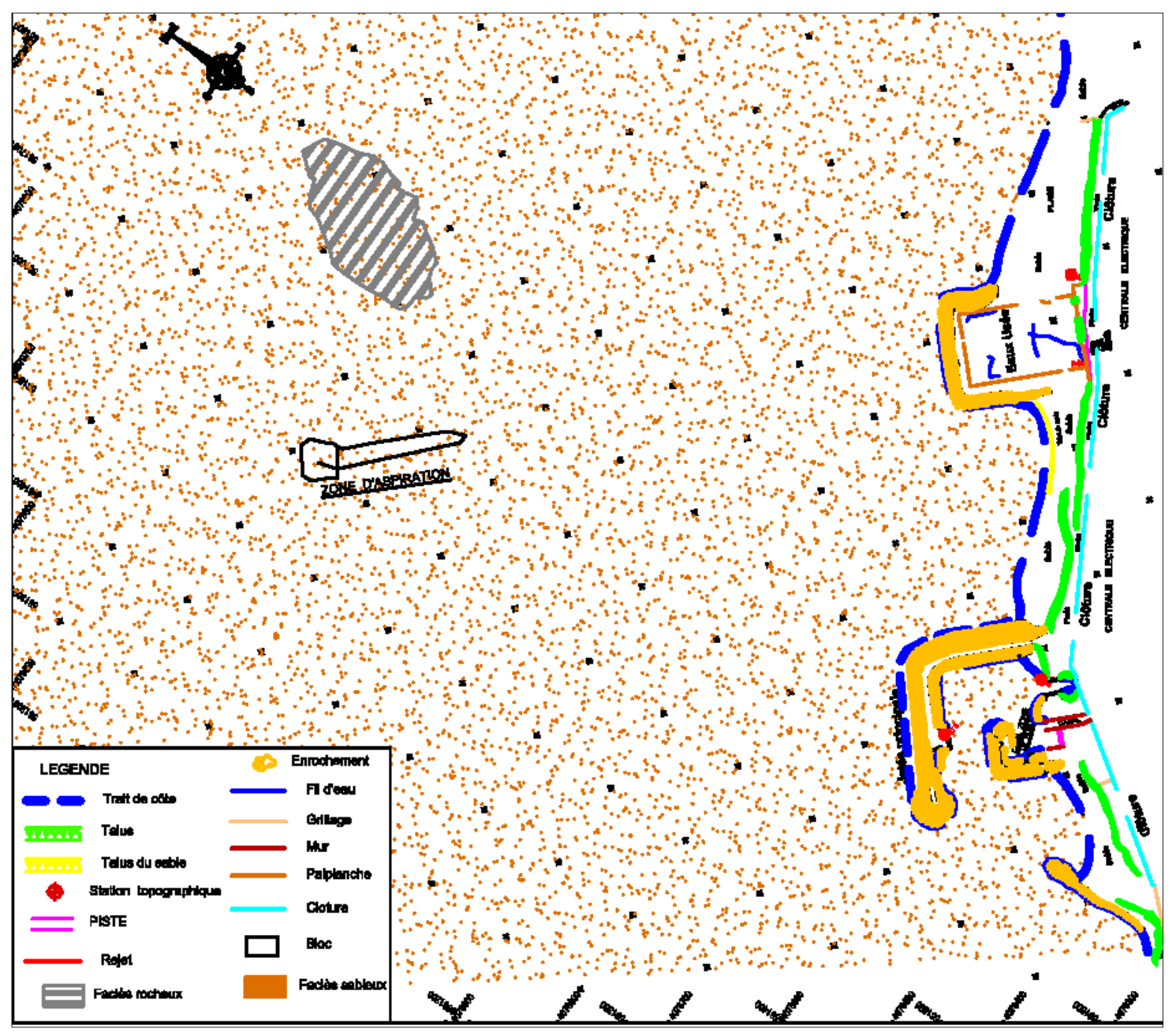

Figure 4. Carte géomorphologique.

\section{Conclusion}

Les résultats de cette étude ont permis de connaître la nature du fond marin et celui du substratum ainsi que la variation des profondeurs qui est en général entre $5 \mathrm{~m}$ et $20 \mathrm{~m}$. Cette étude a également permis de mettre en évidence les différents faciès du fond marin qui est essentiellement sableux.

\section{Références bibliographiques}

CHOUAKI B. (1981). Rapport de reconnaissances préliminaires des sols marins de la centrale thermique de Cap Djinet. Laboratoire d'Etudes Maritimes LEM, 8 p.

FICHEUR M. (1895). Carte géologique de Thénia ex Ménerville à 1/50000. Service géologique de l’Armée Française.

GLANGEAUD L., AYME A., CAIRE A., MATTAUER M., MURAOUR P. (1952). Histoire géologique de la province d'Alger. XIX Cong .Géol. Int., Alger, Mono. Rég., $1^{\circ}$ Série, Algérie, 25, 40 Fig., 3 pl. h.t. 142 p. 